\title{
The deletion of six amino acids at the C-terminus of the $\alpha 1$ (II) chain causes overmodification of type II and type XI collagen: further evidence for the association between small deletions in COL2A1 and Kniest dysplasia
}

\author{
Andreas Winterpacht, Andrea Superti-Furga, Ulrike Schwarze, Hartmut Stöss, Beat
} Steinmann, Jürgen Spranger, Bernhard Zabel

\begin{abstract}
We have identified an $18 \mathrm{bp}$ deletion in exon 49 of the type II procollagen gene (COL2A1) in a patient with Kniest dysplasia. The deletion is located at the very C-terminus of the helical domain and removes two of three Gly-Pro-Pro triplets at positions 1007-1012, which are thought to be involved in helix formation and stability. Morphological investigation of an iliac crest biopsy showed large inclusions in the endoplasmic reticulum of chondrocytes, reflecting impaired secretion of type II collagen. Electrophoretic analysis of collagens extracted from cartilage or synthesised by cultured chondrocytes showed that type II and also type XI procollagen molecules containing mutant $\alpha 1$ (II) chains showed post-translational overmodification. These observations provide further evidence for the general association of Kniest dysplasia with small deletions in the helical domain of type II collagen.
\end{abstract}

(† Med Genet 1996;33:649-654)

Key words: COL2A1; Kniest dysplasia; collagen.

Children's Hospital,

University of Mainz, Langenbeckstrasse 1, D-55101 Mainz,

Germany

A Winterpacht

U Schwarze

J Spranger

B Zabe

Division of Metabolic and Molecular

Diseases, Department of Pediatrics,

University of Zürich, CH-8032-Zürich, Switzerland

A Superti-Furga

B Steinmann

Institute of

Pathology - St

Jonannis Stift, D-33102

Paderborn, Germany

H Stöss

Correspondence to: Dr Winterpacht.

Received 15 September 1995 Revised version accepted fo publication 20 March 1996 have mostly been found to be the result of Gly substitutions in the triple helical domain, with the substituted amino acid and the position of the substitution defining the severity of the phenotype. In contrast, nonsense mutations leading to a premature stop codon were observed in Stickler dysplasia, indicating a quantitative, rather than a structural, collagen II defect as the basis of this disorder. ${ }^{1}$

Kniest dysplasia is a severe form of chondrodysplasia characterised by generalised short trunk dwarfism, kyphoscoliosis, enlarged joints with limited motion, a flat midface, cleft palate, myopia, and hearing loss. The radiographic findings include a characteristic pattern of vertebral, epiphyseal, and metaphyseal abnormalities. ${ }^{2-4}$ Histological and biochemical data suggested that the disorder may be caused by type II collagen defects. ${ }^{5}$ Recently, we have identified the first COL2A1 gene mutation in a patient with Kniest dysplasia: a 28 bp deletion spanning the exon 12 /intron 12 boundary resulted in skipping of exon $12 .^{7}$ In a further case, a point mutation at the $3^{\prime}$ (acceptor) splice site of intron 20 was detected, leading to usage of an alternative splice site in exon 21 and causing a deletion of six amino acids. ${ }^{8}$ In addition, two unrelated patients with identical COL2A1 defects have been reported: they presented with point mutations that created a new preferred $5^{\prime}$ (donor) splice site in exon 12 resulting in a deletion of seven amino acids from the protein. ${ }^{9}$ Only one Gly substitution has been reported in a case of Kniest dysplasia; the mutation was located in exon 12 and changed glycine ${ }_{103}$ to aspartate. ${ }^{10}$

We have now identified a new 18 bp deletion in exon 49 of the COL2A1 gene in a boy with Kniest dysplasia. The deletion is located at the extreme end of the triple helical domain and removed two Gly-Pro-Pro triplets, which are thought to play a critical role in helix stabilisation. We give a detailed description of the molecular defect and the effects on the biochemical level, on chondrocyte morphology, and on the resulting clinical phenotype.

\section{Patient and methods}

THE PATIENT

A 5 year old boy (fig 1) presented with short stature, relatively short trunk with mild pectus excavatum, accentuated lumbar lordosis, and slightly reduced joint mobility. Facial features include flat midface, myopia, cleft palate, and retrognathia. The radiographic findings described in the legend to fig 1 can be 
summarised as irregularities of the ovoid shaped vertebral bodies, flared metaphyses, and small epiphyses.

MOLECULAR ANALYSIS

DNA was extracted from peripheral blood leucocytes. Amplification was carried out with AmpliTaq (Perkin Elmer Cetus), using the following conditions: $50 \mathrm{mmol} / 1 \mathrm{KCl}, 1.5 \mathrm{mmol} / 1$ $\mathrm{MgCl}_{2}, 10 \mathrm{mmol} / 1$ Tris-HCl (pH 8.3), $0.5 \mu \mathrm{g}$ DNA, and $25 \mathrm{pmol}$ of each primer in a total volume of $50 \mu \mathrm{l}$. Amplification was performed after an initial heating to $95^{\circ} \mathrm{C}$ for 30 seconds, for 10 seconds at $95^{\circ} \mathrm{C}$, for 10 seconds at $60^{\circ} \mathrm{C}$, for 10 seconds at $72^{\circ} \mathrm{C}$ over 35 cycles in a Perkin Elmer (system 9600) thermocycler, with a final extension of five minutes at $72^{\circ} \mathrm{C} ; 1 / 10$ volume of the PCR products were analysed on an $8 \%$ polyacrylamide gel. Oligonucleotides for amplification of exon 49 were: upstream 5'-acaatccaggctgatctct-3', downstream 5'caggcccagctctgccetgtactag-3'.

For SSCP analysis, $10 \mathrm{pmol}$ of the primers were end labelled with $\gamma^{32}$ P-ATP $(5000 \mathrm{Ci}$ $\left.\mathrm{mmol}^{-1}\right)$. The PCR reactions were performed as described above, with $2.5 \mathrm{pmol}$ of each labelled primer and $0.25 \mu \mathrm{gNA}$; $1 / 5$ volume of the reaction was separated on a $6 \%$ polyacrylamide gel as described by Orita et al. ${ }^{11}$ Additional bands were cut out of the gel, reamplified, and cloned into plasmid vector pUC18. Sequencing was carried out with the chain termination method ${ }^{12}$ using supercoiled double stranded plasmid DNA as template. ${ }^{13}$
BIOCHEMICAL ANALYSIS OF CARTILAGE COLLAGENS

Cartilage from an iliac crest biopsy performed when the patient was 6 years old was studied. Several cartilage samples from newborns and infants who had died from various causes unrelated to connective tissue disorders were used as control samples. Cartilage was minced finely and extracted with $100 \mu \mathrm{l} / \mathrm{mg}$ wet tissue of a solution containing $4 \mathrm{~mol} / 1$ guanidinium chloride, $50 \mathrm{mmol} / 1 \mathrm{Tris}-\mathrm{Cl}$, and $1 \mathrm{mmol} / \mathrm{l}$ each of phenylmethane-sulphonylfluoride, N-ethylmaleimide, and EDTA, for 48 hours at $8^{\circ} \mathrm{C}$ with continuous stirring. The residue was washed with PBS and further extracted with the same volume of $0.5 \mathrm{~mol} / 1$ acetic acid containing $1 \mathrm{mg} / \mathrm{ml}$ of pepsin at $8^{\circ} \mathrm{C}$ for 48 hours. The pepsin extracts were cleared by centrifugation, and aliquots of the supernatant were lyophilised, resuspended in sample buffer, and separated by SDS-PAGE in 5\% gels containing $0.5 \mathrm{~mol} / 1$ urea under nonreducing conditions. Pepsin resistant collagens were visualised by a silver staining procedure. To study the thermal stability of collagen II extracted from cartilage, aliquots of the pepsin extract were dialysed against $400 \mathrm{mmol} / \mathrm{l}$ $\mathrm{NaCl}, 50 \mathrm{mmol} / \mathrm{l}$ Tris-Cl, $\mathrm{pH} \mathrm{7.4}$, gradually heated, and then incubated with trypsin (final concentration $20 \mu \mathrm{g} / \mathrm{ml}$ ) for two minutes. After incubation with trypsin, the samples were frozen, lyophilised, redissolved in sample buffer, and analysed by SDS-PAGE and silver staining.

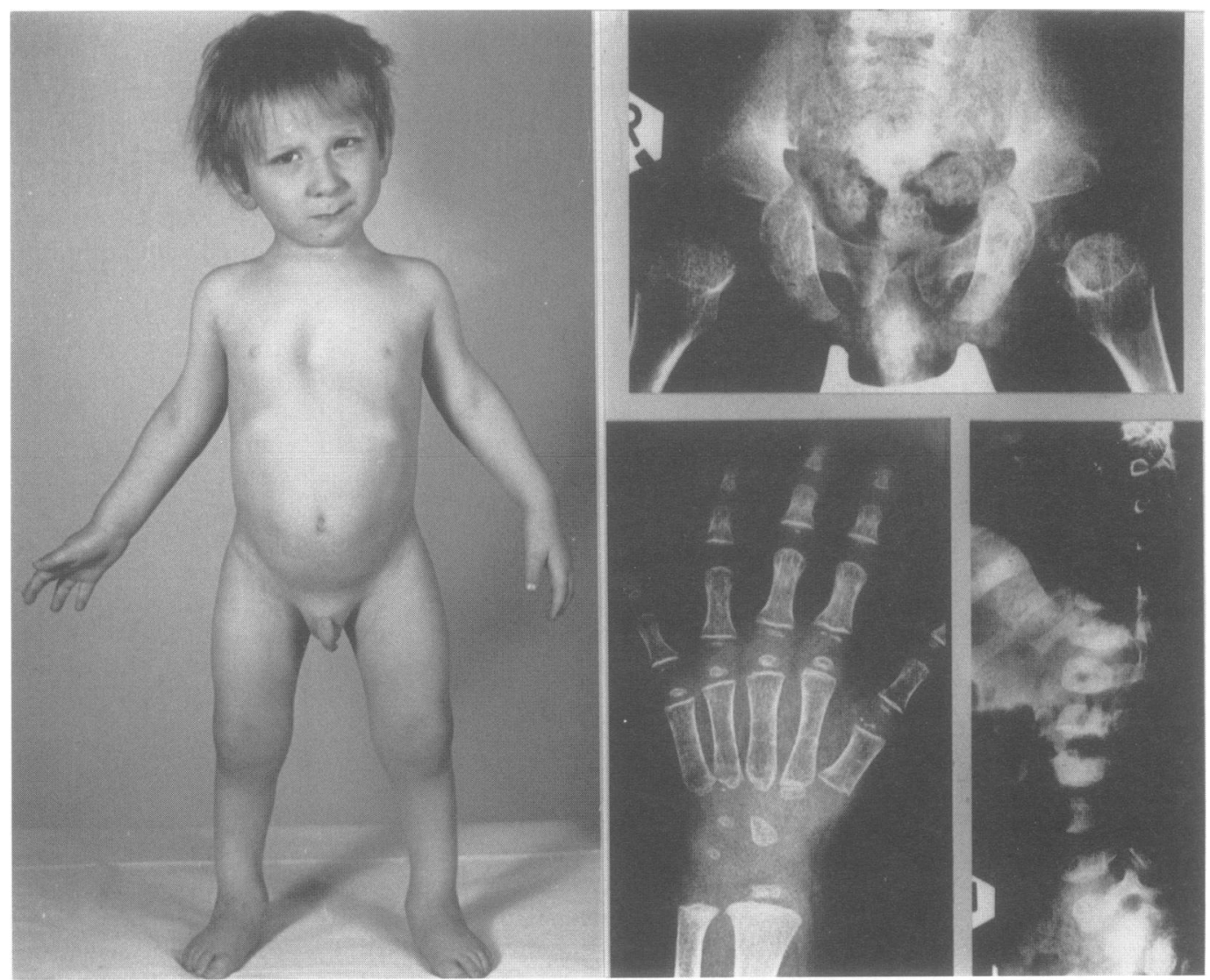

Figure 1 (Left) Patient at the age of 5 years. (Right) $X$ ray findings included slightly ovoid vertebral bodies with some irregularities of the end plates (bottom right). The iliac bodies were broad and flattened. The femora showed an accentuated trochanteric region and very small femoral head epiphyses (top). The tubular bones of the hands showed irregular and mildly flared metaphyses, together with very small and irregular epiphyses (bottom left). 


\section{Chondrocyte isolation}

An aliquot of the cartilage tissue obtained by iliac crest biopsy from the patient, and of control cartilage tissue from femoral heads of other people, was minced finely and incubated overnight in Ham's F1 medium containing antibiotics, $1 \mathrm{mmol} / 1$ cysteine, and $4 \mathrm{mg} / \mathrm{ml}$ of CLS-1 collagenase (Worthington). After dissolution of the tissue, the chondrocytes were collected by centrifugation, washed with culture medium, plated on tissue culture dishes, and allowed to reach confluency. The dedifferenti-

$$
\text { A }
$$

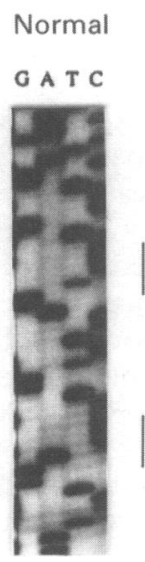

Mutant

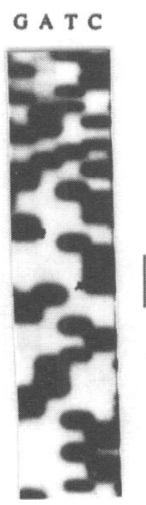

Normal

Aen pro aly Pro Pro Oly Pro Pro Gly Pro Pro Gly Pro Gly Ile

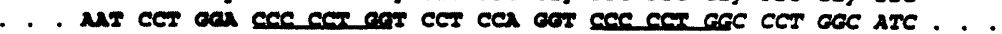

Mutant

Aen pro oly

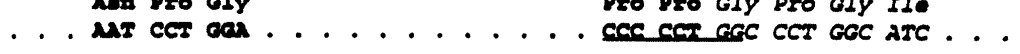

B
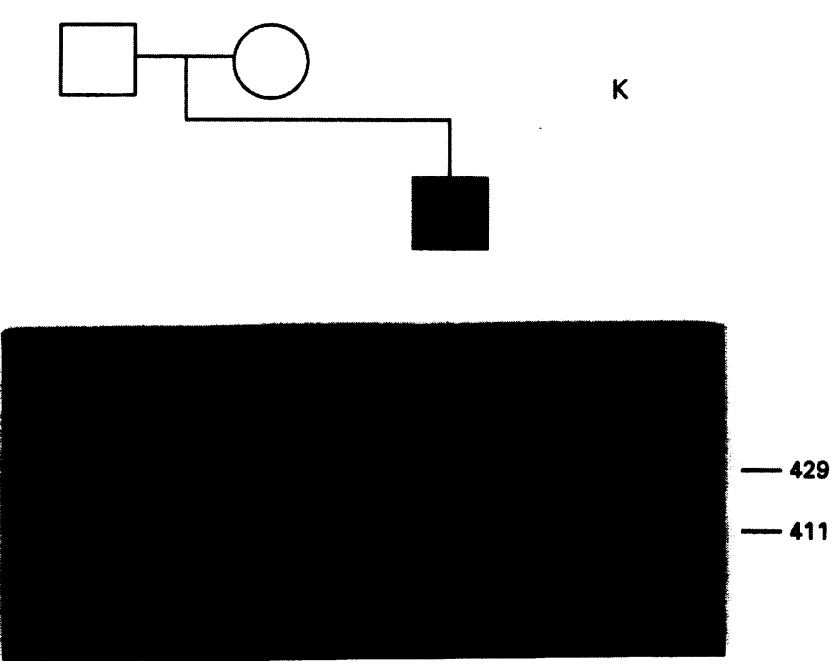

Figure 2 Sequence analysis of normal and mutant exon 49. (A) The 18 bp deletion is located between two $8 \mathrm{bp}$ direct repeats (underlined) and removed two stretches of Gly-Pro-Pro residues. The exact boundaries of the deletion cannot be precisely determined. (B) PCR amplification of exon 49 from the proband, his parents, and an unrelated, unaffected subject (K). In addition to the normal $429 \mathrm{bp}$ product, a mutant $411 \mathrm{bp}$ product, representing the deletion, is only present in the proband. ated chondrocytes were passaged once to increase their number and then either preserved in liquid nitrogen or used directly for biosynthetic studies.

\section{Collagen biosynthesis of chondrocytes cultured on} agarose

To reinduce the chondrocytic phenotype, chondrocytes were plated on tissue culture dishes coated with $0.8 \%$ agarose dissolved in Eagle's Minimal Essential Medium containing $4 \%$ dialysed fetal calf serum (FCS) and 10 $\mathrm{ng} / \mathrm{ml} \mathrm{TGF}-\beta$, and allowed to settle. After 24 hours, the chondrocytes were resuspended by gentle pipetting, centrifuged, and resuspended in cysteine and methionine free MEM containing $4 \%$ dialysed $\mathrm{FCS}$ and $100 \mu \mathrm{Ci} / \mathrm{ml}$ of ${ }^{35} \mathrm{~S}$-methionine (TranSlabel, Flow Laboratories). After overnight incubation, the chondrocytes were resuspended in the labelling medium by gentle pipetting, and the medium containing the chondrocytes was collected, sonicated to disrupt the chondrocytes, and cleared of cell debris by centrifugation. Macromolecules in the supernatant were precipitated by the addition of one half volume of absolute ethanol followed by centrifugation. The pellet was resuspended in $0.5 \mathrm{~mol} / 1$ acetic acid, and aliquots were incubated with pepsin, lyophilised, resuspended in electrophoresis sample buffer, and analysed by SDS-PAGE and autoradiography.

\section{MORPHOLOGICAL ANALYSIS}

An iliac crest biopsy from the patient was fixed in phosphate buffered $4 \%$ paraformaldehyde solution. For histological investigation part of the biopsy was decalcified by $5 \%$ formic acid, embedded in paraffin, and stained with $\mathrm{HE}$, EvG, PAS-alcian blue, and toluidine blue. For electron microscopy the tissue was embedded undecalcified in low viscosity epoxy resin using a modification by Schulz. ${ }^{14}$ Ultrathin sections were contrasted by uranyl acetate and lead citrate.

\section{Results}

MOLECULAR DEFECT

Using a set of 46 primer pairs, which were designed according to the exon/intron boundaries published by Ala-Kokko and Prockop, ${ }^{15}$ we amplified all 54 exons of the COL2A1 gene. The amplification products, which range in size between 113 and $300 \mathrm{bp}$, were screened for the presence of point mutations by single strand conformation polymorphism (SSCP) analysis. " In exon 49 we detected a change leading to an additional band on the SSCP gel. Sequencing of this exon showed an $18 \mathrm{bp}$ deletion at the extreme end of the triple helical domain (fig 2A). The deletion, which does not interrupt the Gly-Xaa-Yaa pattern, most probably occurred by unequal crossing over between two 8 bp direct repeats (fig 2A). Although the exact boundaries cannot be determined precisely, the deletion removed two of three Gly-Pro-Pro triplets considered to be involved in helix formation (see below).

In order to confirm the result, we amplified exon 49 from the patient, his parents (fig $2 \mathrm{~B}$ ), 
and 30 unaffected controls (data not shown). PCR products were separated on an $8 \%$ polyacrylamide gel. The deletion was present exclusively in the genomic DNA of the proband.

A

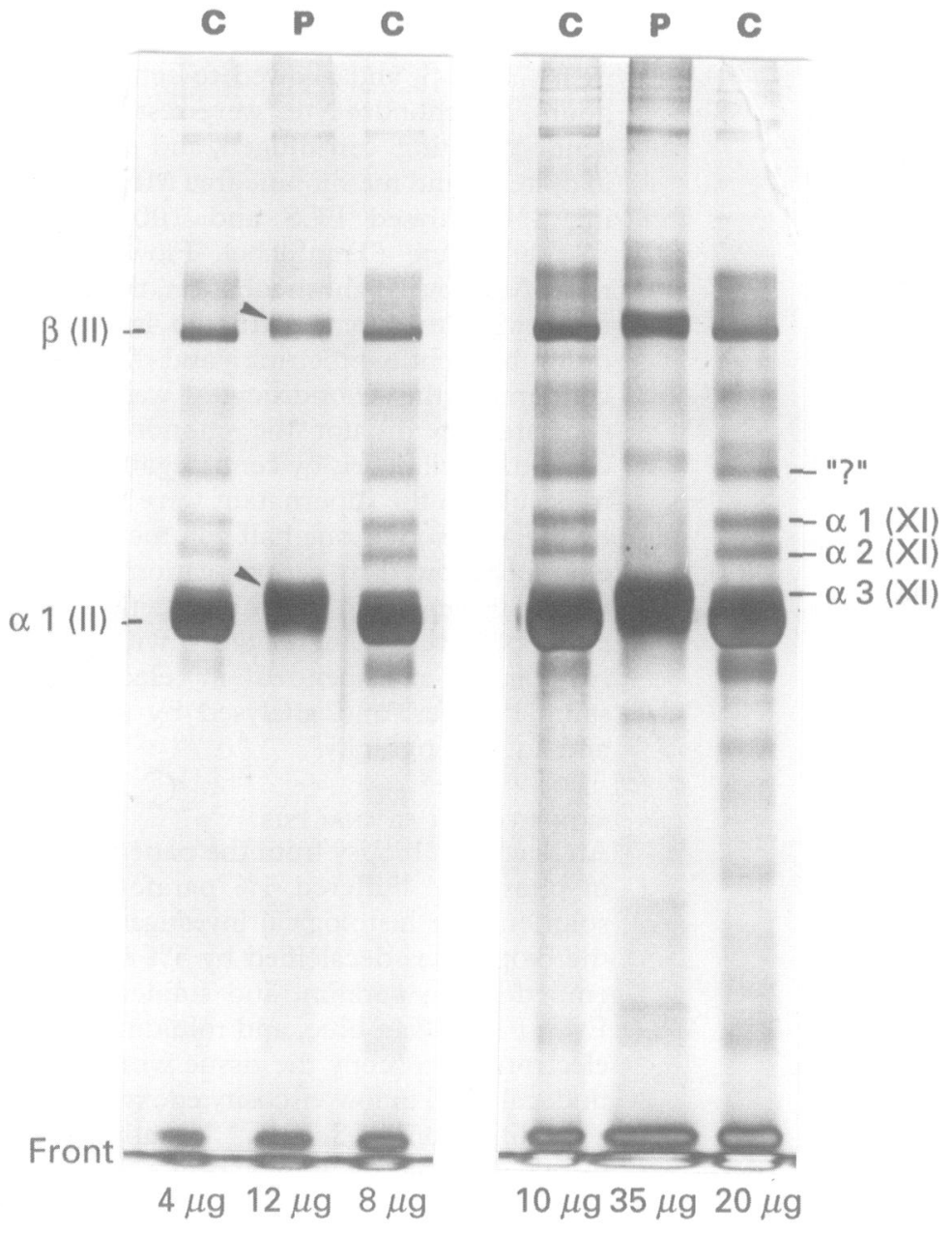

B

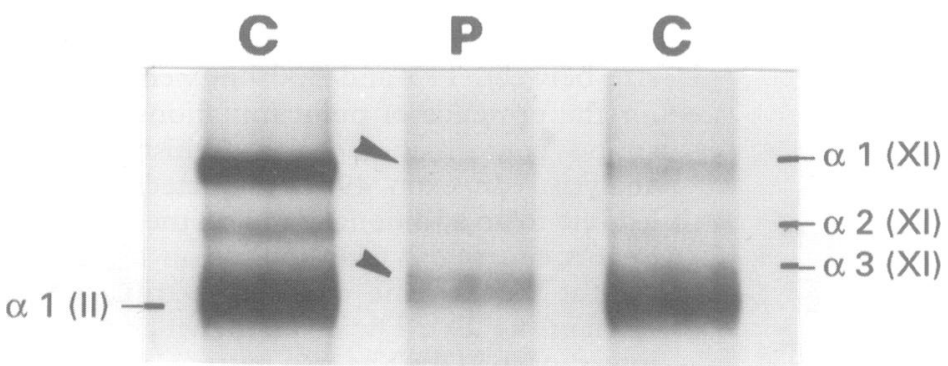

Figure 3 (A) SDS-PAGE analysis of pepsin extracts of cartilage (silver staining). $C$, control samples; $P$, patient's sample. In the patient's sample, the electrophoretic mobility of the $\alpha 1$ (II) and of the dimeric $\beta$ (II) bands is markedly reduced (arrowheads); the $\alpha 1(X I)$ and $\alpha 2(X I)$ bands are faint and migrate slightly slower than their counterparts in the control samples. A further pepsin resistant (and thus probably collagenous) band, marked with "?", is seen above the $\alpha 1$ (XI) band; its migration is also delayed in the patient's sample. The amount of cartilage tissue corresponding to the pepsin extract loaded in each lane is shown at the bottom. (B) Central area of an SDS-PAGE autoradiogram of collagens produced by cultured chondrocytes. Note the decreased mobility of $\alpha 1$ (II), $\alpha 1(X I)$, and $\alpha 2(X I)$ in the patient's sample.
BIOCHEMICAL ANALYSIS

SDS-PAGE analysis of cartilage extracts showed that in the patient with Kniest dysplasia, the collagen II content was moderately reduced (fig $3 \mathrm{~A}$ ) as indicated by the fact that more extract had to be loaded to obtain a band of intensity comparable to that of the controls. Moreover, the mobility of collagen II was reduced (upward shift of the $\alpha 1$ (II) and of the $\beta(\mathrm{II})$ bands, fig $3 \mathrm{~A}$ ); the latter observation would be compatible with substantial posttranslational overmodification. In the sample from the patient with Kniest dysplasia, the $\alpha 1(\mathrm{XI})$ and $\alpha 2(\mathrm{XI})$ bands were relatively faint and their migration was also slightly delayed (fig 3A). Moreover, in all samples an additional, more intense band migrating above $\alpha 1(\mathrm{XI})$ was observed, the mobility of which was also reduced in the patient. The nature of the latter band is not clear (marked "?" in fig $3 \mathrm{~A})$. The thermal stability assay of the patient's cartilage collagens showed that the overmodified collagen II became susceptible to proteolysis around the temperature of $42^{\circ} \mathrm{C}$ and thus did not differ from normally migrating collagen II in the same sample and in control samples (not shown).

The patient's cultured chondrocytes appeared to synthesise reduced amounts of collagens II and XI (fig 3B). Again, the mobility of both the $\alpha 1$ (II) and of the $\alpha 1$ (XI) chains was reduced, although not to the same extent as observed previously in cartilage extracts. The latter observation may be explained by a more pronounced post-translational modification of normal collagen II and collagen XI chains in chondrocyte cultures as compared to the chains synthesised under physiological conditions in cartilage tissue.

MORPHOLOGICAL ANALYSIS

Histological examination of cartilage biopsy material from the patient showed various changes in the growth plate. The columnar organisation of the chondrocytes was disturbed as well as the complete hypertrophic zone. Small areas of the extracellular matrix showed a dispersed organisation (Swiss cheese-like appearance), which is quite characteristic of Kniest dysplasia cartilage. ${ }^{56916}$ Large cytoplasmic inclusions were visible in the chondrocytes. Immunohistochemical analysis showed that these inclusions reacted strongly with type II collagen antibodies, in contrast to a slight reaction in the surrounding matrix, indicating reduced excretion of type II collagen from the chondrocytes (data not shown). In addition, electron microscopy showed collagen fibres that varied extremely in diameter. Areas with plump, broad fibrils with splayed ends alternated with areas of very thin, kinked fibres, mainly in the growth plate (fig 4). Moreover, large dilatations in the rough endoplasmic reticulum of the chondrocytes were visible and contained a fine granular material (fig 4D).

\section{Discussion}

In the present study we describe an $18 \mathrm{bp}$ deletion in exon 49 at the very C-terminus of the collagenous domain of type II procollagen in a 
patient with Kniest dysplasia. As the mutation was not found in the patient's parents or in unaffected controls, it can be regarded as the cause of the disorder in this patient. Morphological examination of the proband's cartilage showed large inclusions with markedly dilated,

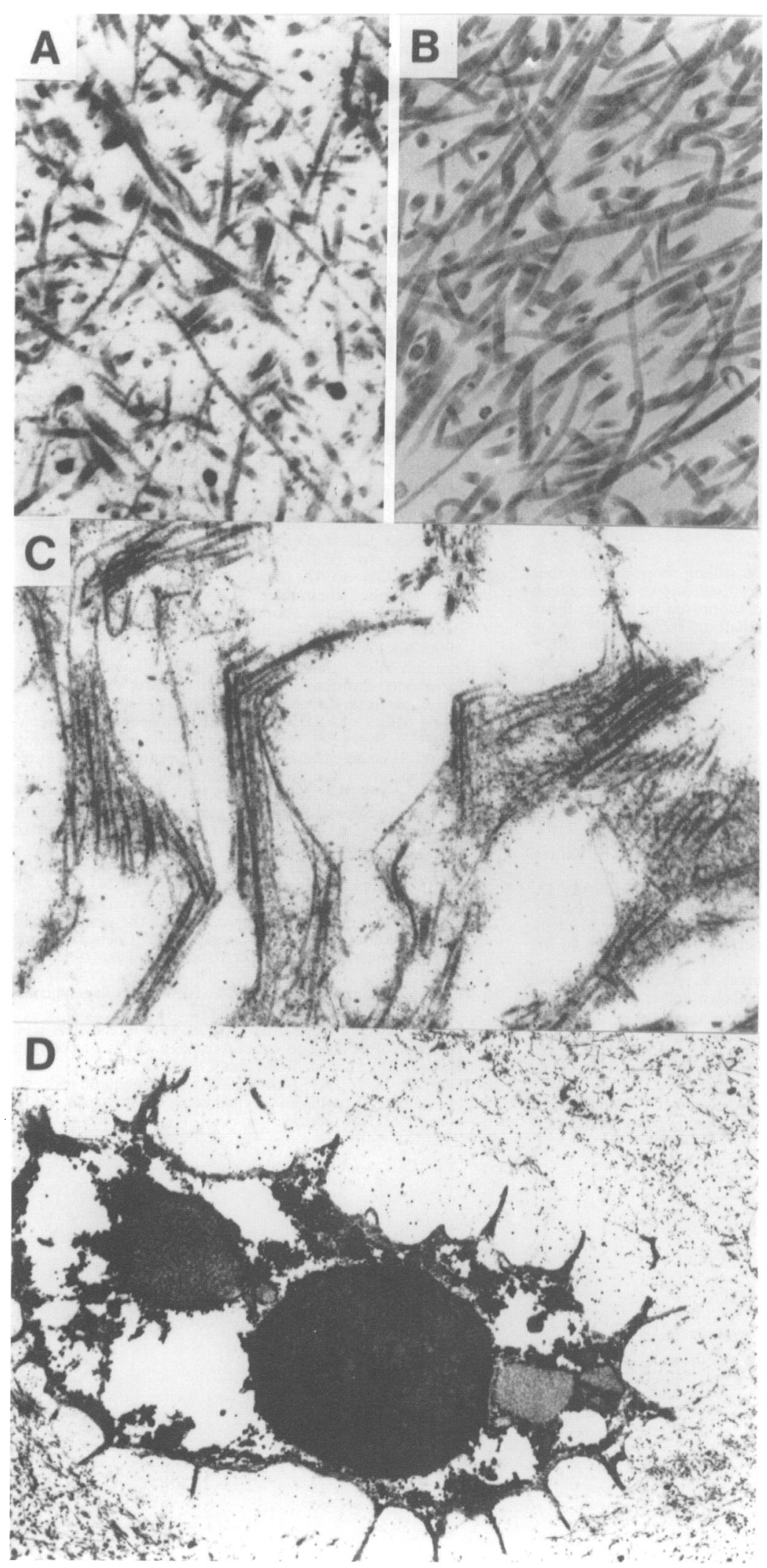

Figure 4 Electron microscopy of the iliac crest biopsy. (A) Extracellular matrix with irregular, plump, broad fibrils, partly splayed ends, varying diameters (EM), and (B) a corresponding normal control tissue. (C) Strongly disorganised collagen fibrils with very thin, kinked fibres (EM). (D) Chondrocyte with large dilated cisternae of the rough endoplasmatic reticulum containing a fine granular material (EM). rough endoplasmic reticulum containing granular material, which stains with type II collagen antibodies. Moreover, disorganised cartilage with sparsely distributed collagen and fibrils highly variable in diameter were visible by light and electron microscopy. The histological findings resemble those described by Horton and Rimoin, ${ }^{16}$ Poole et al, ${ }^{5}$ and Bogaert et $a l,{ }^{9}$ and confirmed the diagnosis of Kniest dysplasia. Protein analysis showed a fraction of slow migrating collagen type II in material extracted directly from cartilage, as well as in collagen isolated from chondrocyte cultures of the proband. Taken together, histological and biochemical data suggest that collagen containing defective chains shows delayed folding within and secretion from the chondrocytes. Delayed migration of mutant collagen is most probably a result of post-translational overmodification and indicates a delay in helix formation. This has been noted in a variety of mutations, including small deletions in the types I, II, and III collagens, ${ }^{9}{ }^{10}{ }^{17-20}$ and directly shown in a series of glycine-to-cysteine substitutions in the $\alpha 1$ (I) chains. ${ }^{21}$ Our results may confirm the hypothesis that misalignment of charged residues causes delayed helix formation, as observed in overmodified collagen I caused by large or small deletions which do not change the Gly-X-Y pattern of the triple helix. $^{22}{ }^{23}$ In the present case, the deletion removed two out of three Gly-Pro-Pro triplets at the end of the triple helical domain. It is known from thermal stability experiments that this triplet combination forms the most stable triple helix and it is widely believed that this region serves as a nucleation point of the different chains before helix folding. ${ }^{24}{ }^{25}$ As a result, the deletion might inhibit or delay triple helix formation right at the beginning, so that the collagen chains become highly overmodified. Although type II collagen is retained in the chondrocytes, some mutant proteins must have been secreted, leading to disorganised cartilage and a highly variable collagen fibril diameter.

The $\alpha 3(\mathrm{XI})$ collagen chain is believed to be a post-translationally modified variant of the $\alpha 1$ (II) collagen chain. ${ }^{26}{ }^{27}$ It has therefore been suggested that COL $2 \mathrm{~A} 1$ gene defects may determine changes in type XI collagen as well. ${ }^{10}$ We were able to show a slight overmodification of the type XI collagen in cartilage and in cultured chondrocytes from our proband. These results represent the first direct evidence that type XI collagen may also be affected by COL2A1 gene mutations. It has been shown that type XI collagen is buried in the interior of the fibrils and may be important in regulating cartilage collagen fibril assembly, ${ }^{28}$ or in determining the diameter of the collagen fibrils, ${ }^{27}$ or both. Therefore, the disturbed matrix and highly variable fibril diameter in the cartilage of our proband may be the result of reduced amounts or structural alterations of both type II and type XI collagen.

So far, six COL2A1 mutations have been found in patients with Kniest dysplasia: a deletion of exon $12,{ }^{7}$ a deletion of six amino acids from exon $21^{8}$ and from exon 34 (Winterpacht 
et al, in preparation), an out of register deletion (disrupting the Gly-Xaa-Yaa pattern) of seven amino acids in two unrelated patients, ${ }^{9}$ and a $\mathrm{Gly}_{103} \rightarrow$ Asp exchange in exon $12 .^{10}$ Taken together, the data suggest that most of the Kniest dysplasia cases are caused by small deletions in the COL2A1 gene (in or out of register). Incorporation of shortened $\alpha$ chains into the fibrils should lead to misalignment of cross linking sites and should therefore interfere with normal intermolecular cross linking, which may explain some specificities of the clinical picture. The Gly $\rightarrow$ Asp exchange in exon 12 described by Wilkin et $a l^{10}$ may be an exception to the rule of an in frame deletion as the specific basic defect, but it supports a special role of exon 12 in the development of the Kniest dysplasia phenotype. Perhaps exon 12 contains an as yet unidentified functional domain, the disruption of which might lead to the Kniest phenotype. ${ }^{10}$ In order to understand fully the relationship between a given COL2A1 mutation and the resulting Kniest phenotype, additional information is needed about interand intramolecular interactions of abnormal collagens and about possible functional domains in fibril forming collagens.

We are grateful to the family for their willing cooperation in this We are grateful to the family for their willing cooperation in this technical assistance. The work was supported by a grant from the Deutsche Forschungsgemeinschaft to BZ and AW, by a the Deutsche Forschungsgemeinschaft to BZ and AW, by a grant from the Swiss National Foundation to ASF (3236387.92 and $32-45401.35)$ and to BS (32-42138.34)), and by
a grant from the European Community to BZ, AW, and ASF.

1 Spranger J, Winterpacht A, Zabel B. The type II collagenopathies: a spectrum of chondrodysplasias. Eur $\mathcal{F}$ Pediatr 1994;153:56-65.

2 Kniest W. Zur Abgrenzung der Dysostosis enchondralis von der Chondrodystrophie. $Z$ Kinderheilkd 1952;70:633-70.

3 Maroteaux P, Spranger J. La maladie de Kniest. Arch Fr Pediatr 1973;30:735-50.

4 Lachman RS, Rimoin DL, Hollister DW, et al. The Kniest syndrome. $A \mathcal{F}$ 1975;123:805-14.

5 Poole AR, Pidoux I, Reiner A, Rosenberg L, Hollister D, Rimoin D. Kniest dysplasia is characterized by an apparent abnormal processing of the C-propeptide of type II cartilage collagen resulting in imperfect fibril assembly. $\mathcal{F}$ Clin lage collagen resulting

6 Poole AR, Rosenberg L, Murray L, Rimoin D. Kniest dysplasia: a probable type II collagen defect. Pathol Immunopathol Res 1988;7:95-8.

7 Winterpacht A, Schwarze U, Mundlos S, Menger $H$, Spranger J, Zabel BU. Kniest and Stickler dysplasia phenotypes caused by collagen type II gene (COL2A1) defect. Nature Genet 1993;3:323-6.

8 Winterpacht A, Hilbert $M$, Schwarze U, Mundlos $S$ Spranger J, Zabel B. Alternative splicing as the result of a type II procollagen gene (COL2A1) mutation in a patient with Kniest dysplasia. Hum Mol Genet 1994;3:1891-3.

9 Bogaert R, Wilkin D, Wilcox WR, et al. Expression in cartilage of a 7-amino-acid deletion in type II collagen from two unrelated individuals with Kniest dysplasia. Am $\mathcal{F}$ Hum Genet 1994;55:1128-36.

10 Wilkin DJ, Bogaert R, Lachman RS, Rimoin DL, Eyre DR, Cohn DH. A single amino acid substitution (G103D) in the type II collagen triple helix produces Kniest dysplasia. Hum Mol Genet 1994;3:1999-2003.

11 Orita M, Suzuki Y, Sekiya T, Hayashi K. Rapid and sensitive detection of point mutations and DNA polymorphisms using the polymerase chain reaction. Genomics 1989; using the

12 Sanger F, Nickeln AR, Coulsen AR. DNA sequencing with chain-terminating inhibitors. Proc Natl Acad Sci USA chain-terminating

13 Chen EY, Seeburg PH. Supercoil sequencing, a fast and simple method for sequencing plasmid DNA. DNA 1985; 4:165-70

14 Schulz A. A reliable method of preparing undecalcified human bone biopsies for electron microscopic investigation. Microscopia Acta 1977;80:7-18.

15 Ala-Kokko L, Prockop DJ. Completion of the intron-exon structure of the gene for human type II procollagen
(COL2A 1): variations in the nucleotide sequences of the alleles from three chromosomes. Genomics 1990;8:454-60.

16 Horton WA, Rimoin DL. Kniest dysplasia. A histochemical study of the growth plate. Pediatr Res 1970;13:1266-70.

17 Chessler SD, Byers PH. Defective folding and stable association with protein disulfide isomerase/prolyl hydroxylase of type I procollagen with a deletion in the proo2(I) chain that preserves the Gly-X-Y repeat pattern. $\mathcal{F}$ Biol Chem 1992;267:7751-7.

18 Wallis GA, Kadler KE, Starman BJ, Byers PH. A tripeptide deletion in the triple-helical domain of the pro $\alpha 1$ (I) chain of type I procollagen in a patient with lethal osteogenesis imperfecta does not alter cleavage of the molecule by N-proteinase. F Biol Chem 1992;267:25529-34.

19 Superti-Furga A, Steinmann B, Ramirez F, Byers PH. Molecular defects of type III procollagen in Ehlers-Danlos Molecular defects of type III procollagen in Eh

20 Cole WG, Chiodo AA, Lamande SR, et al. A base substitution at a splice site in the COL3A1 gene causes exon skipping and generates abnormal type III procollagen in a patient with Ehlers-Danlos syndrome type IV. $\mathcal{F}$ Biol Chem 1990;265:17070-7.

21 Ragunath M, Bruckner P, Steinmann B. Delayed triple helix formation of mutant collagen from patients with osteogenesis imperfecta. $\mathcal{F}$ Mol Biol 1994;236:940-9.

22 Willing MC, Cohn DH, Starman B, Holbrook KA, Greenberg CR, Byers PH. Heterozygosity for a large deletion in the $\alpha 2(I)$ collagen gene has a dramatic effect on type I colhe $\alpha 2(1)$ coliagen genesis imperfecta. $¥$ Biol Chem 1988;263:8398-404.

23 Hawkins JR, Superti-Furga A, Steinmann B, Dalgleish R. A 9-base pair deletion on COL1A1 in alethal variant of osteogenesis imperfecta. $\mathcal{F}$ Biol Chem 1991;266:22370-4.

24 Doege KJ, Fessler JH. Folding of carboxyl domain and assembly of procollagen I. $\mathcal{F}$ Biol Chem 1986;261:8924-35.

5 Kielty CM, Hopkinson I, Grant ME. The collagen family: structure, assembly, and organization in the extra-cellular matrix. In: Royce PM, Steinmann B, eds. Connective tissue and its heritable disorders. New York: Wiley Liss, 1993:10348.

26 Vuorio E, de Crombrugghe B. The family of collagen genes. Annu Rev Biochem 1990;59:837-72.

27 Mendler M, Eich-Bender S, Vaughan L, Winterthalter $\mathrm{KH}$, Bruckner P. Cartilage contains mixed fibrils of collagen types II, IX, and XI. $₹$ Cell Biol 1989;108:191-7.

28 Thom JR, Morris NP. Biosynthesis and proteolytic processing of type IX collagen in embryonic chick sterna. $\mathcal{F}$ Biol Chem 1991;266:7262-9. 